\title{
Nonlinear rheology of dense colloidal dispersions: a phenomenological model and its connection to mode coupling theory
}

\author{
Matthias Fuchs 1 , 困 and Matthias Ballauff ${ }^{2}$ \\ ${ }^{1}$ Fachbereich Physik, Universität Konstanz, 78457 Konstanz, Germany \\ ${ }^{2}$ Physikalische Chemie I, Universität Bayreuth, 95440 Bayreuth, Germany
}

(Dated: November 15, 2018)

\begin{abstract}
Rheological properties, especially 'shear-thinning', of dense colloidal dispersions are discussed on three different levels. A generalized phenomonological Maxwell model gives a broad framework connecting glassy dynamics to the linear and non-linear rheology of dense amorphous particle solutions. First principles mode coupling theory calculations for the time or frequency dependent shear modulus give quantitative results for dispersions of hard colloidal spheres in the linear regime. Schematic models extending mode coupling theory to the non-linear regime recover the phenomenology of the generalized Maxwell model, and predict universal features of flow curves, stress versus shear-rate.

PACS numbers: 82.70.Dd, 83.60.Df, 83.50.Ax, 64.70.Pf, 83.10.-y
\end{abstract}

\section{INTRODUCTION}

The rheological behavior of dispersions of rigid colloidal particles is immensely rich [1, 2]. Universally they exhibit shear-thinning, that the viscosity $\eta$ decreases from the Newtonian-plateau upon increasing the shear rate $\dot{\gamma}$. Another phenomenon in dense colloidal dispersions, or dispersions of particles with strong attractions, is that they undergo arrest-transitions and solidify to (mostly weak) amorphous solids (glasses) [3, 4, 5, 6, 7, 8, 9]. In this contribution, we want to establish under which circumstances and simplifications one may expect that the physics of the glass transition determines or even dominates the rheological behavior of colloidal dispersions. Our discussion supplements a recent comparison of theoretical computations and measurements of flow curves in colloidal model systems where this connection is explored quantitatively [10].

While recently microscopic approaches to shearthinning in colloidal dispersions have been presented [11, 12, 13, 14], in this contribution we first argue on a more qualitative level and sketch a phenomenological picture, that goes back to Maxwell. Then, we discuss in how far simple 'schematic models' derived from the first principles approaches in Refs. 11, 12, 13, 14], support the generalized phenomenological Maxwell model. The concepts we discuss build upon insights into the structural dynamics obtained with the mode coupling theory (MCT) of Götze and coworkers 15, 16], and thus extend a microscopic approach which has amply been tested and compared to computer simulation studies and experimental data from quiescent model systems [17]. We also present MCT predictions for the linear viscoelasticity of colloidal hard spheres which may be tested in future simulational or rheological studies.

\footnotetext{
${ }^{*}$ Corresponding author. Email: matthias.fuchs@uni-konstanz.de
}

\section{A PHENOMENOLOGICAL GENERALIZED MAXWELL MODEL}

\section{A. Quiescent system}

Before discussing the connection of glassy dynamics with the rheology of dense colloidal dispersions, it appears helpful to reconsider the oldest phenomenological description of the glass transition which goes back to Maxwell. Maxwell considered the most general constitutive equation relating (transversal) stress $\sigma$ and shear rate $\dot{\gamma}$ within the framework of linear response theory:

$$
\sigma(t)=\int_{-\infty}^{t} d t^{\prime} G^{\mathrm{tot}}\left(t-t^{\prime}\right) \dot{\gamma}\left(t^{\prime}\right) .
$$

Here, $G^{\text {tot }}(t)$ is the shear modulus and is, within linear response, independent of the applied shear rate $\dot{\gamma}$, which in the most general situation is a time-dependent function. This relation is exact for the linear response of systems with free energy corresponding to a fluid phase provided that the system was in equilibrium before the (smoothly switched-on) application of shear 18]. For colloidal solutions, Batchelor was the first to discuss the three contributions to the stress which arise: in the solvent, from the potential interactions among the particles, and from the solvent particle interactions [19]. For the dispersions of interest here, the solvent can be considered a continuum fluid characterized by a know solvent viscosity $\eta_{s}$, so that $G^{\text {solv }}(t)=\eta_{s} \delta(t)$.

Solvent-particle interactions (viz. the HI) act instantaneously if the particle microstructure differs from the equilibrium one, but do not themselves determine the equilibrium structure. If one assumes that glassy arrest is connected with the ability of the system to explore its configuration space and to approach its equilibrium structure, then it appears natural to assume that the solvent particle interactions are characterized by a finite time scale $\tau_{\mathrm{HI}}$. In a most naive way, the contribution from HI, $G^{\mathrm{HI}}(t)$, to the shear modulus $G^{\text {tot }}(t)$ would thus be written as: $G^{\mathrm{HI}}(t)=G_{\infty}^{\mathrm{HI}} \exp \left\{-t / \tau_{\mathrm{HI}}\right\}$, where the 
time $\tau_{\mathrm{HI}}$ stays finite and varies little with the distance to glassy arrest. Considering time-independent shear rates, $\dot{\gamma}(t)=\dot{\gamma}$, so that

$$
\sigma=\eta \dot{\gamma}=\dot{\gamma} \int_{0}^{\infty} d t G^{\mathrm{tot}}(t)
$$

HI would thus lead to an increase of the high frequency viscosity above the solvent value; this value shall be denoted as $\eta_{\infty}=\eta_{s}+G_{\infty}^{\mathrm{HI}} \tau_{\mathrm{HI}}$.

Maxwell in his studies of viscous liquids considered the potential contribution, to be abbreviated as $G(t)$, to the shear modulus, which arises from the forces between the particles. Its microscopic definition is, $G(t)=$ $\left\langle\sigma_{x y}(t) \sigma_{x y}(0)\right\rangle /\left(k_{B} T V\right)$, where the relevant element of the fluctuating stress tensor is given with the potential forces $\mathbf{F}_{i}$ and particle coordinates $\mathbf{r}_{i}$ by $\sigma_{x y}(t)=$ $-\sum_{i} F_{i}^{x}(t) y_{i}(t)$. Kinetic contributions that would differ between Newtonian (which Maxwell considered) and Brownian (the colloidal dispersions considered here) systems do not contribute for symmetry reasons. He postulated that close to the glass transition the modulus develops a slow relaxational process that is characterized by a time scale $\tau$, which grows beyond bounds:

$$
G(t)=G_{\infty} \exp \left\{-\frac{t}{\tau}\right\} .
$$

The amplitude of the slow relaxational process, $G_{\infty}$, can be considered an almost constant that changes much more slowly with density or other parameters than the time scale $\tau$. The divergence of $\tau$ upon solidification often is modeled [2] by a Krieger-Dougherty, $\tau / \tau_{0} \propto$ $\left(1-\phi / \phi_{m}\right)^{-2}$, or a Doolitle form, $\ln \tau / \tau_{0} \propto\left(1-\phi / \phi_{m}\right)^{-1}$. Here the packing fraction at the maximal fluid density is denoted as $\phi_{m}$, and the time-unit $\tau_{0}$ should be connected to the viscosity $\eta_{\infty}$ that a particle feels because of the surrounding solvent. The slow process described by Maxwell, which in the glass literature is called $\alpha$-process, causes a marked and strongly density dependent increase of the total viscosity over $\eta_{\infty}$ : performing the integration gives $\eta_{0}=\eta_{\infty}+G_{\infty} \tau$. In this picture, the total shear modulus as a function of time consists of the three mentioned terms: $G^{\mathrm{tot}}(t)=\eta^{s} \delta(t)+G^{\mathrm{HI}}(t)+G(t)$.

The naive picture sketched here, is not correct for a number of reasons. It is well known that for hard spheres without HI the shear modulus diverges for short times, $G^{\mathrm{HSnoHI}}(t \rightarrow 0) \sim t^{-1 / 2}$. Lubrication forces, which keep the particles apart, eliminate this divergence and ren$\operatorname{der} G^{\text {tot }}(t \rightarrow 0)$ finite [20]. Thus, the simple separation of $G^{\mathrm{HI}}(t)$ and the potential $G(t)$ is not possible for short times, at least for particles with a hard core. Moreover, comparison of simulations without and with $\mathrm{HI}$ has shown that the increase of $\left(\eta_{0}-\eta_{\infty}\right) / \eta_{\infty}$ depends somewhat on HI, and thus not just on the potential interactions as implied.

Nevertheless the sketched picture provides the most basic view of a glass transition in colloidal suspensions, connecting it with the increase of the structural relaxation time $\tau$. Increased density or interactions cause a slowing down of particle rearrangements which leave the HI relatively unaffected, as these solvent mediated forces act on all time scales. Potential forces dominate the slowest particle rearrangements because vitrification corresponds to the limit where they actually prevent the final relaxation of the microstructure. The structural relaxation time diverges at the glass transition, while $\tau^{\mathrm{HI}}$ stays finite. Thus close to arrest a time scale separation is possible, $\tau \gg \tau^{\mathrm{HI}}$.

While the parameter $G_{\infty}$ in Maxwell's model might appear to play a rather boring role as amplitude of the final relaxation process, its actual significance is quite high. It gives the elastic constant of the arrested structure in the limit of $1 / \tau=0$ (viz. in the glass), or of the glassy structure present on intermediate times, $t \ll \tau$. Thus in the glass or for shear rates $\dot{\gamma}(t)$ that vary more rapidly with time than the modulus, a Hookian elastic response follows from Eq. (11):

$$
\begin{aligned}
& \sigma(t)=G_{\infty} u(t)+\text { dissipative terms, } \quad \text { where } \\
& u(t)=\int_{-\infty}^{t} d t^{\prime} \dot{\gamma}\left(t^{\prime}\right) \quad \text { for } 1 / \tau=0
\end{aligned}
$$

where $u(t)$ is the strain imposed on the amorphous solid. Clearly, $G_{\infty}$ plays the role of a spring constant for the elastic restoring forces in the solid which grow with the imposed distortion measured by the strain, viz. $u(t)=$ $\int^{t} d t^{\prime} \partial v_{x}\left(t^{\prime}\right) / \partial y$.

An interesting difference from this hall mark behavior of glasses to colloidal coagulation or gelation at low packing fractions should be noted. Experiments on a wide range of gels at high dilutions have shown that the elastic constant $G_{\infty}$ vanishes at the colloidal gelation line; it then grows with a strong power-law upon entering deeper into the gel phase [21]. It has been pointed out that this behavior can be explained by an underlying percolation like transition. Thus the observed 'Trappe-scaling' points to a deep difference between colloidal coagulation at very low packing fractions and colloidal vitrification at higher ones. At the latter transition, $G_{\infty}$ is finite, as first postulated by Maxwell. Interestingly, this difference does not show up in universal jamming phase diagrams. Intriguing data have been obtained for intermediate concentration range where both pictures transform, but further studies appear very fruitful 22].

The elastic constant $G_{\infty}$ serves to indicate a difference of the present Maxwell's view on the glass transition to another colloid theory. It is the approach by Brady [23], who identifies the solidification of amorphous dispersions with random close packing, $\phi_{m}=\phi_{\text {rcp }}$. In this approach, the modulus $G^{\text {tot }}$ relaxes in a single relaxational process characterized by the time scale $\tau^{\mathrm{s}}=a^{2} / D_{0}^{s} ; a$ is the particle radius, and $D_{0}^{s}$ the density dependent short-time self diffusion constant. While Brady's final result for the viscosity, $\eta_{0} \propto\left(1-\phi / \phi_{m}\right)^{-2}$, is similar to glassy behavior (MCT predicts a divergence $\eta_{0} \propto \tau \propto\left(1-\phi / \phi_{c}\right)^{-2.6}$ for hard spheres), part of the divergence of $\eta_{0}$ comes from a divergence of the elastic constant upon approaching random close packing, $G_{\infty} \propto\left(1-\phi / \phi_{m}\right)^{-1}$. The 
time scale $\tau^{\mathrm{s}}$ also diverges at solidification, but contributes only comparable to the amplitude of the relaxational process, $\tau^{\mathrm{s}} \propto\left(1-\phi / \phi_{m}\right)^{-1}$. Thus, a quite different scenario of arrest at random close packing to arrest at the glass transition should become apparent if $G^{\text {tot }}(t)$ or its Fourier-transforms, elastic $G^{\prime}(\omega)$ and loss $G^{\prime \prime}(\omega)$ moduli are measured. (We use the convention $\left.G^{\prime \prime}(\omega)+i G^{\prime}(\omega)=\omega \int_{0}^{\infty} d t e^{i \omega t} G(t)\right)$ While at the glass transition the dynamics should only become slower, at random close packing the amplitude of the stress at intermediate times should also increase. Besides this qualitative difference, a quantitative difference also arises in the values of arrest, namely the glass transition density, called $\phi_{c}$ in MCT and measured in, lies below random close packing: $\phi_{c} \approx 0.58<\phi_{r c p} \approx 0.64$ 3, 4, [5, 6, 7, 8]. Particles in a glass thus still have local volume to rearrange; they can explore their respective 'cages', but cannot relax the overall microstructure.

\section{B. Under shear}

The utility of the phenomenological Maxwell model of the glass transition is further stressed by the ease by which it can be extended to the shear-thinning of colloidal fluids and yielding of colloidal glasses under shear. The central concept required is that shearing speeds up the relaxation of fluctuations and kills off the long-time memory present in very viscous fluids. This requires that shear causes the slow structural or $\alpha$-relaxational process in $G(t)$ of the Maxwell model to decay faster. It is natural to connect the relaxation time of the structural relaxation to the inverse of the shear rate, and thus to formulate on the phenomenological level:

$$
g^{\mathrm{gMm}}(t, \dot{\gamma})=G^{\mathrm{tot}}(t) \exp \{-t|\dot{\gamma}|\} .
$$

Here, the generalized non-linear shear modulus $g(t, \dot{\gamma})$ is a function describing the transient fluctuations in a dispersion sheared from time $t=0$ on. Equation (6) expresses that under shear transient stress fluctuations either decay via the relaxation channels present without shear, or via a shear induced relaxation mechanism whose intrinsic decay rate is set by the shear rate itself. Postulating that the shear rate enters in a non-analytic way via the absolute value into the phenomenological description is of course, a priori, questionable, and requires the derivation given in Refs. 11, 12]; see also section IV. The result Eq. (6) is quite satisfying, because, intuitively, one expects the direction of the solvent flow profile to be irrelevant for the magnitude of the stress or viscosity even in the non-linear regime.

The generalization of Eq. (11), that connects $g(t, \dot{\gamma})$ to the steady state stress in the case of constant shearing is:

$$
\begin{aligned}
\sigma(\dot{\gamma}) & =\eta(\dot{\gamma}) \dot{\gamma}=\dot{\gamma} \int_{0}^{\infty} d t g^{\mathrm{gMm}}(t, \dot{\gamma}) \\
& =\dot{\gamma}\left\{\eta_{s}+\frac{\eta_{\infty}-\eta_{s}}{1+|\dot{\gamma}| \tau_{\mathrm{HI}}}+\frac{\eta_{0}-\eta_{\infty}}{1+|\dot{\gamma}| \tau}\right\} .
\end{aligned}
$$

This is the final expression of the extended Maxwell model for the stationary viscosity or stress as a function of the shear rate, $\eta(\dot{\gamma})$. Variations of the packing fraction or of other control parameters of the system without shear enter via the Maxwell relaxation time $\tau$, and consequently $\eta_{0}$, which may for example be modeled by a Krieger Dougherty expression below the glass transition density, and by $\tau=\infty=\eta_{0}$ above it. All other constants appearing explicitly or implicitly in Eq. (7) may, in the most simple approach, be taken as control-parameter independent constants.

The discussed phenomenology is quite rich with flow curves that exhibit $(i)$ a Newtonian viscosity plateau given by $\eta_{0}$ at low shear rates and below the glass transition (for $|\dot{\gamma}| \ll 1 / \tau \ll 1 / \tau_{\mathrm{HI}}$ ), (ii) a shear thinning region for $1 / \tau \ll|\dot{\gamma}| \ll 1 / \tau_{\mathrm{HI}}$ below the glass transition, (iii) a solid-like yield flow for $0=1 / \tau \ll|\dot{\gamma}| \ll 1 / \tau_{\mathrm{HI}}$ above the glass transition, and (iv) a second Newtonian plateau characterized by $\eta_{\infty}$ following the shear thinning region while still $|\dot{\gamma}| \ll 1 / \tau_{\mathrm{HI}}$ holds. In this region of high shear, where the HI are dominant, the suggested modeling of $G^{\mathrm{HI}}(t)$ is not based on theoretical insights from our microscopic approach, and thus is not reliable. Therefore, the most sensible approach, that we also will follow in the remainder of this presentation, is to restrict the shear rates to the region $|\dot{\gamma}| \tau_{\mathrm{HI}} \ll 1$. Then Eq. (7) simplifies to $\eta(\dot{\gamma})=\eta_{\infty}+\left(\frac{1}{\eta_{0}}+\frac{|\dot{\gamma}|}{\sigma^{*}}\right)^{-1}$, which captures the small shear rate limit close to vitrification, where $\tau \gg \tau_{\mathrm{HI}}$, and $\eta_{0} \gg \eta_{\infty}$. The notation $\sigma^{*}$ has been introduced, to stress the dimensionalities; from Eq. (7) $\sigma^{*}=G_{\infty}$ follows, while in more general settings $\sigma^{*}$ is more closely related to the critical yield stress at solidification [14].

Aim of section IV is to present and discuss a schematic model, which can be derived within our microscopic approach [1], 12], and which recovers the phenomenology just described.

\section{VISCOELASTICITY OF QUIESCENT COLLOIDAL FLUIDS}

Mode coupling theory (MCT) appears to capture the cage-effect and predicts that it dominates the slow relaxation of structural correlations [16]. Density fluctuations play an important role because they are well suited to describe the structure of the particle system and its relaxation. Moreover, stresses that decay slowly because of the slow particle rearrangements, MCT argues, also can be approximated by density fluctuations using effective potentials. Density fluctuations not at large wavelengths, but for wavelengths corresponding to the average particle distance turn out to be the dominant ones. In agreement with the picture of the caging of particles by structural correlations, the MCT glass transition is independent on whether the particles move ballistically in between interactions with their neighbors (say collisions for 
hard spheres) or by diffusion. Structural arrest happens whenever the static density correlations for wavelengths around the average particle distance are strong enough. Thus, it had been realized early on that this basic version of MCT, the 'idealized MCT', that was developed for a description of the structural arrest in atomic systems also needs to apply to colloidal dispersions [16].

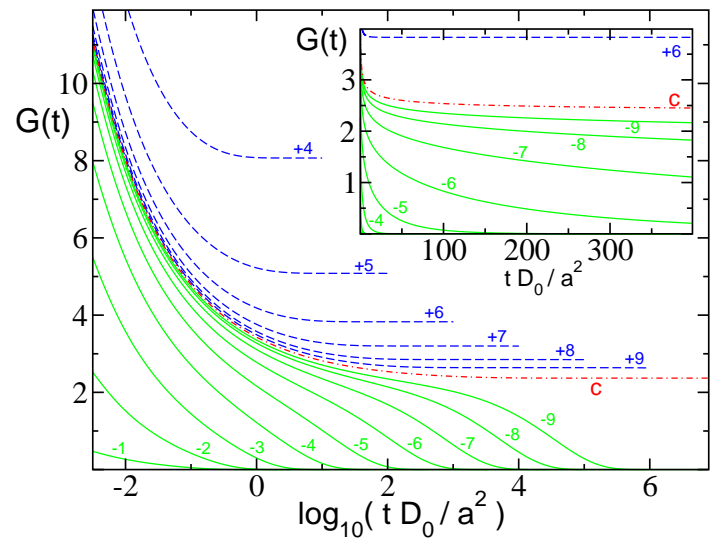

FIG. 1: Equilibrium potential shear modulus $G(t)$ (in units of $\left.k_{B} T / a^{3}\right)$ for colloidal hard spheres with radius $a$ for packing fractions close to solidification at $\phi_{c}$. Densities are measured by the separation parameter $\varepsilon=\left(\phi-\phi_{c}\right) / \phi_{c}= \pm 10^{-|n| / 3}$, and labels denote the value $n$. Positive values belong to glass $(\varepsilon>0)$, negative to fluid states $(\varepsilon<0)$; the label $c$ gives the transition. The inset shows a subset of the curves on a linear time axis; the increase of $G(t)$ for short times cannot be resolved.

Results of the (idealized) MCT equations for the potential part of the time-dependent shear modulus $G(t)$ of hard spheres for various packing fractions $\phi$ are shown in Fig. 1] and calculated from [15]:

$$
G(t) \approx \frac{k_{B} T}{60 \pi^{2}} \int_{0}^{\infty} d k k^{4}\left(\frac{\partial \ln S_{k}}{\partial k}\right)^{2} \Phi_{k}^{2}(t)
$$

The normalized density fluctuation functions $\Phi_{k}(t)=$ $\left\langle\delta \varrho^{*}(\mathbf{k}, t) \delta \varrho(\mathbf{k})\right\rangle /\left\langle|\delta \varrho(\mathbf{k})|^{2}\right\rangle /$ enter, which are calculated self-consistently within MCT 25]. Packing fractions are measured in relative separations $\varepsilon=\left(\phi-\phi_{c}\right) / \phi_{c}$ to the glass transition point, which for this model of hard spheres lies at $\phi_{c}=0.516[15]$. Note that this result depends on the static structure factor $S(q)$ only, which is taken from Percus-Yevick theory, and that the experimentally determined value $\phi_{c}^{\text {expt. }}=0.58$ lies somewhat higher [3, 5]. For low packing fractions, or large negative separations, the modulus decays quickly on a time-scale set by the short-time diffusion of well separated particles. The strength of the modulus increases strongly at these low densities, and its behavior at short times presumably depends sensitively on the details of hydrodynamic and potential interactions; thus Fig. 1 is not continued to small times, where the employed model (taken from Ref. 24]) is too crude. For smaller (negative) separations from the critical density, $\varepsilon \nearrow 0$, little changes in $G(t)$ at short times, because the absolute change in density becomes small. Yet, at long times a process in $G(t)$ becomes progressively slower upon taking $\varepsilon$ to zero. It can be considered the MCT analog of the phenomenological Maxwell-process. MCT finds that it depends on the equilibrium structural correlations only, while HI and other short time effects only shift its overall time scale. Importantly, this overall time scale applies to the slow process in coherent and incoherent density fluctuations as well as in the stress fluctuations 25. This holds even though e.g. HI are known to affect short time diffusion coefficients and high frequency viscosities differently. Upon crossing the glass transition bifurcation, the relaxation freezes out and the amplitude $G_{\infty}$ of the Maxwell-process does not decay; the modulus for long times approaches the elastic constant of the glass $G(t \rightarrow \infty) \rightarrow G_{\infty}>0$. Entering deeper into the glassy phase the elastic constants increase quickly with packing fraction.

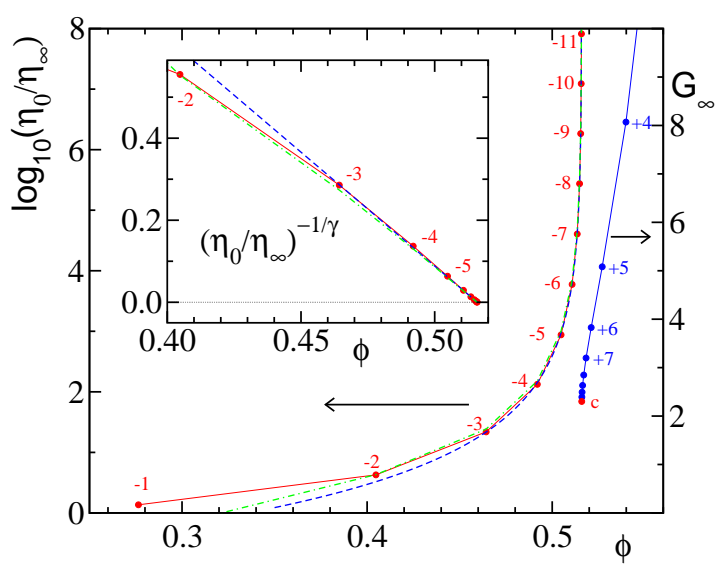

FIG. 2: On the left side, increase of the Newtonian low frequency shear viscosity $\eta_{0}$ relative to the high frequency one, $\eta_{\infty}$, for hard spheres as function of the packing fraction $\phi$; numbers (negative as $\phi<\phi_{c}$ ) denote the separation $\varepsilon=\left(\phi-\phi_{c}\right) / \phi_{c}= \pm 10^{-|n| / 3}$. Neglecting hydrodynamic interactions, $\eta_{\infty}=\frac{k_{B} T}{6 \pi D_{0} a}$ is obtained from the short time diffusion coefficient. The dashed line gives the asymptotic power law $\left(\phi_{c}-\phi\right)^{\gamma}$ predicted by MCT with $\gamma=2.46$, while the relaxation times $\tau_{\eta}$ obtained from $G\left(\tau_{\eta}\right)=k_{B} T /(2 a)^{3}$ are multiplied by a factor $0.2^{-\gamma}$ and shown by a dot-dashed line. The inset presents the same data in a rectification plot, $\left(\eta_{0} / \eta_{\infty}\right)^{-1 / \gamma}$, where the power-law becomes a straight line. On the right side, the long time limits of the shear modulus, $G(t \rightarrow \infty)=G_{\infty}$ in units of $k_{B} T / a^{3}$, are shown versus $\phi$, with positive numbers $n$ denoting the separation from $\phi_{c}$.

Calculating the viscosity according to Eq. (2), $\eta_{0}=$ $\eta_{\infty}+\int_{0}^{\infty} d t G(t)$ it is important to realize that the integration is dominated by the slow process. The inset of Fig. 1 highlights this by showing $G(t)$ on a linear axis of time. The viscosity increases as the area under $G(t)$, which is almost exclusively set by the slowest process, which is the Maxwell one. The rapid decrease of $G(t)$ to the plateau value $G_{\infty}$ does not affect the viscosity as long as time-scale separation holds and the Maxwell process 
is much slower. Figure 2 exhibits the obtained increase of the low frequency viscosity $\eta_{0}$ for a quiescent colloidal dispersion of hard spheres. Also shown is the asymptotic power law predicted by MCT (with prefactor fitted), and the variation of the longest relaxation time $\tau_{\eta}$ of $G(t)$ estimated by $G\left(\tau_{\eta}\right)=k_{B} T /(2 a)^{3}$. Asymptotically all three quantities exhibit the same dependence on $\phi$, and diverge at the glass transition density $\phi_{c}$. The asymptotic power law variation holds for relative separations up to around $-\varepsilon \approx 10 \%$.

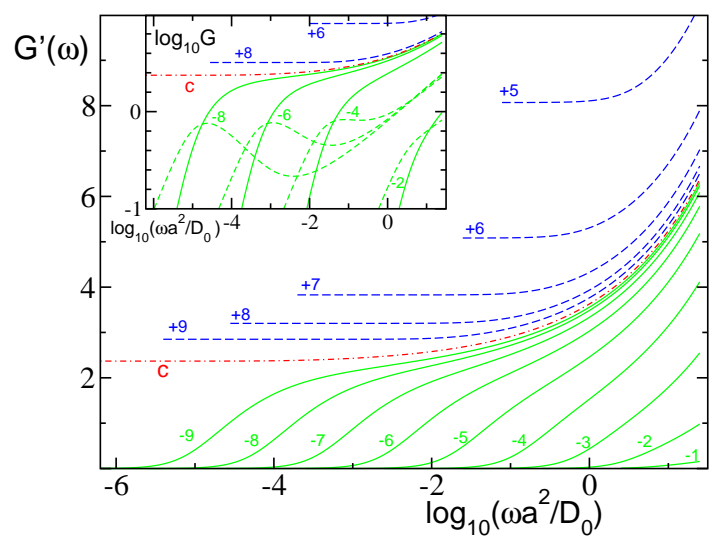

FIG. 3: Storage part of the shear modulus $G^{\prime}(\omega)$ corresponding to Fig. 11 The inset shows storage and loss moduli (only for fluid states) for a number of densities.

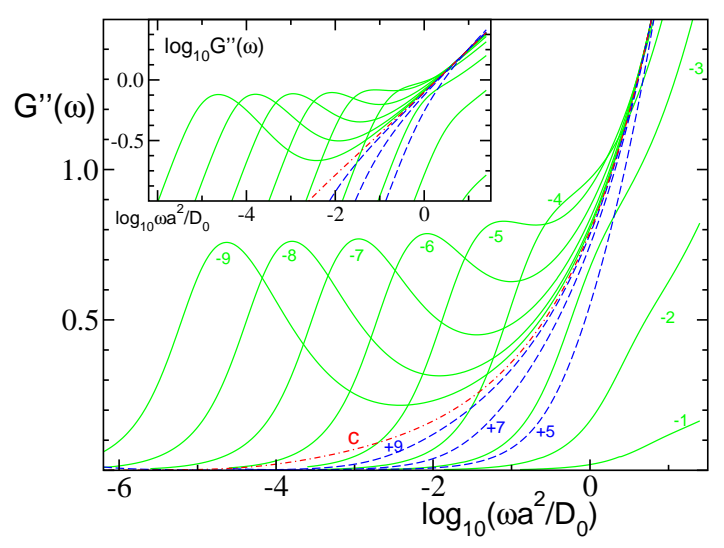

FIG. 4: Loss part of the shear modulus $G^{\prime \prime}(\omega)$ corresponding to Fig. 11 The inset shows the same data in a double logarithmic representation.

MCT thus provides a microscopic description of the linear viscoelasticity that follows the phenomenology first presented by Maxwell; of course in detail important and interesting differences exist, e.g. the exponential shape of the final decay postulated by Maxwell is replaced by a stretched-exponential decay in MCT. Tests of these specific predictions have greatly contributed to the verification of MCT [17].

While $G(t)$ can be accessed by theory more directly, experimentally its Fourier-transforms can be studied more easily. Storage $G^{\prime}(\omega)$ and loss $G^{\prime \prime}(\omega)$ modulus are shown as functions of frequency in Figs. 3 and 4 respectively. The slow Maxwell-process appears as a shoulder in $G^{\prime}$ which extends down to lower and lower frequencies when approaching glassy arrest, and reaches to zero frequency in the glass, $G^{\prime}(\omega=0)=G_{\infty}$. The slow process shows up as a peak in $G^{\prime \prime}$ which in parallel motion (see inset of Fig. 3) shifts to lower frequencies when $\varepsilon \nearrow 0$. Including, in the spirit of the discussion in Sect.II, hydrodynamic interactions into the calculation would affect the frequency dependent moduli at frequencies satisfying $\omega \tau_{\mathrm{HI}} \approx 1$. For smaller frequencies, only a small correction would arise, $G_{\mathrm{HI}}^{\prime \prime}\left(\omega \ll 1 / \tau_{\mathrm{HI}}\right) \sim \omega \eta_{\infty}$ and $G_{\mathrm{HI}}^{\prime}\left(\omega \ll 1 / \tau_{\mathrm{HI}}\right) \sim\left(\omega \eta_{\infty}\right)^{2} / G_{\infty}^{\mathrm{HI}}$, as long as time scale separation and consequently $\eta_{0} \gg \eta_{\infty}$ holds.

\section{SCHEMATIC MODELS FOR THE NON-LINEAR RHEOLOGY}

While within the MCT the linear viscoelastic regime has been worked out quantitatively, the recent generalization of MCT to the non-linear regime [1, 12] has not yet been fully solved. Only a number of universal predictions have been obtained, which exist in any model that exhibits the generic bifurcation scenario from yielding solid to shear thinning fluid. In this section we verify that this scenario recovers the phenomenological Maxwell picture discussed in Sect. II. Hereto we use the most simple schematic model that has also recently been successfully employed to analyse flow curves from simulations [13, 26] and experiments [10].

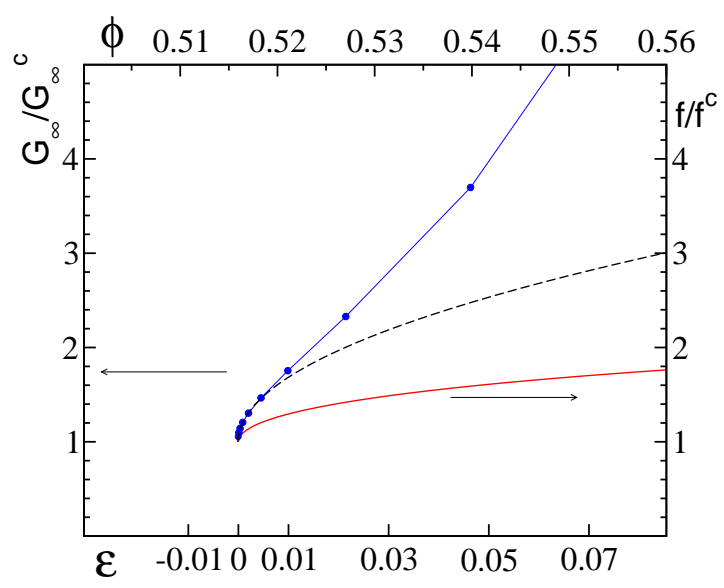

FIG. 5: Normalized frozen-in glass parameters for a microscopic model, viz the elastic constant of a hard sphere glass $G_{\infty} / G_{\infty}^{c}$, and a schematic model, viz. the non-ergodicity parameter $f / f_{c}$ of the $F_{12}$-model, as functions of the separation parameter $\varepsilon$; the normalization is the respective value at the transition. The upper axis gives $\varepsilon$ expressed in packing fractions for hard spheres.

The central feature of the equations of motion in Refs. [1, 12] is that they contain the competition of two effects. First, a non-linear memory effect increases with 
increasing particle interactions ('collisions' or 'cage effect') which leads to a non-ergodicity transition in the absence of shear, and second, memory effects vanish with time because of shear-induced decorrelation. Both these effects can be captured in the simpler 'schematic' models also. Importantly, the models can be made to obey similar stability equations as the microscopic approach, and thus the universal predictions hold.

The well studied and comparatively simple schematic $\mathrm{F}_{12}^{(\dot{\gamma})}$-model considers one normalized correlator $\Phi(t)$, which obeys a generalized relaxation equation:

$$
\dot{\Phi}(t)+\Gamma\left\{\Phi(t)+\int_{0}^{t} d t^{\prime} m\left(t-t^{\prime}\right) \dot{\Phi}\left(t^{\prime}\right)\right\}=0 .
$$

Without memory effects, $m \equiv 0$, the correlator relaxes exponentially, $\Phi(t)=\exp -\Gamma t$, but with $m \neq 0$, retardation effects set in after a short-time variation (still given by the initial decay rate $\Gamma$, viz. $\Phi(t \rightarrow 0)=1-\Gamma t+\ldots)$. The correlator $\Phi(t)$ may be thought to correspond to the normalized shear modulus of the generalized Maxwell model of section II. A low order polynomial ansatz for $m$ suffices to model the feedback mechanism of the cageeffect. We choose

$$
m(t)=\frac{1}{1+(\dot{\gamma} t)^{2}}\left(v_{1} \Phi(t)+v_{2} \Phi^{2}(t)\right) .
$$

Without shear, this model has been studied extensively [15, 27]. Increasing particle caging is modeled by increasing coupling parameters $v_{1}, v_{2} \geq 0$, and the only effect of shearing is to cause a time dependent decay of the friction kernel $m$. The system loses memory because of shearing. The role of the transport coefficient (viscosity) $\eta$ is played by the average relaxation time obtained from integrating the correlator, and this also is taken to determine the stress:

$$
\sigma=\dot{\gamma} \eta=\dot{\gamma}\langle\tau\rangle=\dot{\gamma} \int_{0}^{\infty} d t \Phi(t) .
$$

At high shear rates, the memory function is strongly supressed, so that $\Phi$ returns to a single exponential, and the high shear visosity of the model follows as $\eta_{\infty}=1 / \Gamma$.

For the parameters of the model, values studied in the literature shall be taken [12, 15, 27]. The parameter $\Gamma$ sets the time scale and determines the short time dynamics. The bare Peclet number for the model thus is given by $\mathrm{Pe}_{0}=\dot{\gamma} / \Gamma$. Following earlier studies, the two interaction parameters are chosen as $v_{2}=v_{2}^{c}=2$ and $v_{1}=v_{1}^{c}+\varepsilon /\left(\sqrt{v_{2}^{c}}-1\right)$, where $v_{1}^{c}=v_{2}^{c}\left(\sqrt{4 / v_{2}^{c}}-1\right) \approx 0.828$. Thus, a dependence on temperature or packing fraction enters the model only via $\varepsilon$. A glass transition singularity lies at $\varepsilon=0$, where the long time limit $\Phi(t \rightarrow \infty)=f$ jumps discontinuously from zero for $\varepsilon<0$ to a finite value $f \geq f_{c}=1-1 / \sqrt{v_{2}^{c}}$ for $\varepsilon \geq 0$ and $\dot{\gamma}=0$. The parameter $f$ plays the role of the elastic constant $G_{\infty}$ in this model.

Comparing the amplitude $f$ of the yielding process in the $F_{12}^{(\dot{\gamma})}$-model with the elastic constants $G_{\infty}$ calculated

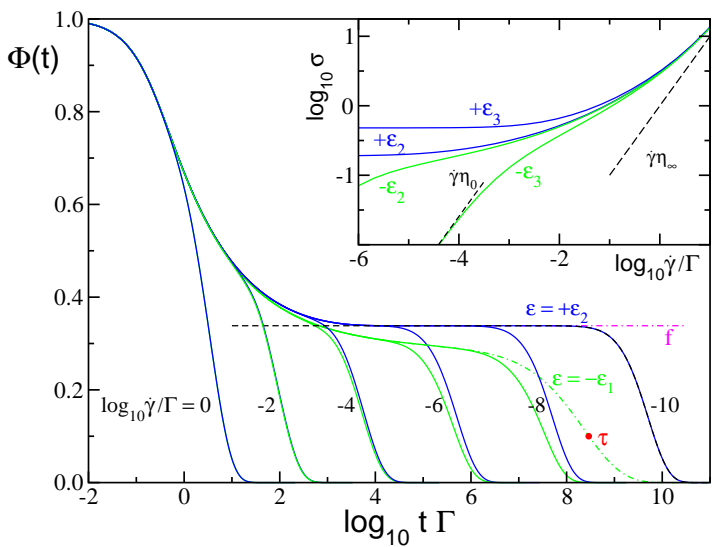

FIG. 6: Correlators of the schematic $F_{12}^{(\dot{\gamma})}$-model as function of rescaled time $t \Gamma$. The curve marked with a relaxation time $\tau$ taken at $\Phi(\tau)=0.1$ corresponds to a fluid state without shear, $\varepsilon=-\varepsilon_{1}=-10^{-3.791}$ and $\dot{\gamma}=0$. For increasing shear rates, $\log _{10}(\dot{\gamma} / \Gamma)=-8,-6,-4,-2,0$, the correlators decay more quickly. The curve marked by the long time plateau value $f$ corresponds to a glass state without shear, $\varepsilon=\varepsilon_{2}=16 \varepsilon_{1}$ and $\dot{\gamma}=0$. For nonvanishing shear rates, $\log _{10}(\dot{\gamma} / \Gamma)=-10,-8$, $-6,-4,-2,0$, the correlators decay more quickly to zero. The scaling function $\phi^{+}$, whose integral gives the yield stress, is shown by a dashed curve shifted onto the $\varepsilon_{2}$ and $\dot{\gamma} / \Gamma=10^{-10}$ curve.

The inset shows flow curves of the model, viz. dimensionless stress $\sigma$ versus $\dot{\gamma} / \Gamma$, for two states that would be fluid respectively glassy without shear; $\varepsilon= \pm \varepsilon_{2}$ and $\varepsilon= \pm \varepsilon_{3}= \pm 16 \varepsilon_{2}$. Straight lines with slope unity indicate the variation following the low shear $\eta_{0}$ and high shear viscosity $\eta_{\infty}$.

for hard spheres in Sect. II.B, one notices a quantitative difference; see Fig. 5 While a change of $\varepsilon$ by $10 \%$ causes an increase in $G_{\infty}$ by a factor 8 , in the schematic model $f$ changes only by $15 \%$. Fitting experimental flow curves of particles with strong repulsive interactions [10] thus the present $F_{12}^{(\dot{\gamma})}$-model can be expected to underestimate the increase of the stress values in the glassy region.

The presence of a glassy arrested structure is equivalent to a frozen in part in the correlator or memory function; thus without shear $\Phi(t \rightarrow \infty)=f>0$ and $m(t \rightarrow \infty)=g>0$ hold for $\varepsilon \geq 0$. With shear a nondecaying part in $m(t)$ is impossible, as $m(t \dot{\gamma} \gg 1) \leq$ $\left(v_{1}+v_{2}\right) /(\dot{\gamma} t)^{2}$; as a consequence, also $\Phi(t)$ always decays to zero. Memory is cut off at long times, and Eq. (10) gives the most simple ansatz recovering this effect of shear advection in the microscopic equations, and the obviously required symmetry in $\dot{\gamma}$. Figure 6 shows for fluid and glassy, at $\dot{\gamma}=0$, solutions of the $F_{12}^{(\dot{\gamma})}$-model the speed up of the relaxation caused by increasing shear rates. Integrating over the correlators, as given in Eq. (11), leads to the viscosity which consequently exhibits shear thinning; representative flow curves are shown in the inset of Fig. [6]

It is interesting to analyse the origin of a yield stress more closely in the schematic model. This can be done most easily at the glass transition point, where the cor- 
relator arrests at the (critical) value of plateau at long times, $\Phi(t \rightarrow \infty)=f_{c}$ at $\varepsilon=0$ and $\dot{\gamma}=0$; this quantity may be interpreted as the elastic constant $G_{\infty}^{c}$ of the amorphous solid at melting. Applying shear, and assuming that the unique steady state has been reached after waiting sufficiently, the schematic model predicts a finite (critical) yield stress $\sigma_{c}^{+}=\int_{0}^{\infty} d x \Phi_{c}^{+}(x)$, that follows from the final decay of the (elastic) constant of the glass. The existence of a yield stress thus is seen to arise from the final relaxational process in the transient, which is only caused by shearing and whose relaxation time is given by $1 /|\dot{\gamma}|$. The phenomenology of the generalized Maxwell model of Sect. II.A is thus recovered in the schematic model, and can be recognized in Fig. 6]
The part frozen in without shear of magitude $f$, decays during a process driven by and characterized by the same time scale as the external shear rate.

\section{Acknowledgments}

We thank T. Franosch, J.-L. Barrat, J. Bergenholtz, L. Berthier, A. Latz and G. Petekidis for discussions. M.F. thanks M.E. Cates with whom the theoretical approach was developed for enlightening discussions. M. B. was supported by the DFG, SFB 481, Bayreuth.
[1] W. B. Russel, D. A. Saville, and W. R. Schowalter, Colloidal Dispersions (Cambridge University Press, New York, 1989).

[2] R. G. Larson, The Structure and Rheology of Complex Fluids (Oxford University Press, New York, 1999).

[3] W. van Megen and S. M. Underwood, Phys. Rev. Lett. 70, 2766 (1993); 72, 1773 (1994).

[4] W. van Megen and S. M. Underwood, Phys. Rev. E 47, 248 (1993).

[5] W. van Megen and S. Underwood, Phys. Rev. E 49, 4206 (1994).

[6] C. Beck, W. Härtl, and R. Hempelmann, J. Chem. Phys. 111, 8209 (1999).

[7] E. Bartsch, T. Eckert, C. Pies, and H. Sillescu, J. NonCryst. Solids 307-310, 802 (2002).

[8] T. Eckert and E. Bartsch, Faraday Discuss. 123, 51 (2003).

[9] T. G. Mason and D. A. Weitz, Phys. Rev. Lett. 75, 2770 (1995).

[10] M. Fuchs and M. Ballauff, J. Chem. Phys., 122, in press (2005).

[11] M. Fuchs and M. E. Cates, Phys. Rev. Lett. 89, 248304 (2002).

[12] M. Fuchs and M. E. Cates, Faraday Disc. 123, 267 (2003).

[13] M. Fuchs and M. E. Cates, J. Phys.: Cond. Mat. 15, S401 (2003).

[14] M. E. Cates, K. Kroy, W. C. K. Poon, A. M. Puertas, and
M. Fuchs, J. Phys.: Condens. Matter 16, S4861 (2004).

[15] W. Götze, in Liquids, Freezing and Glass Transition, edited by J.-P. Hansen, D. Levesque, and J. Zinn-Justin (North-Holland, Amsterdam, 1991), p. 287.

[16] W. Götze and L. Sjögren, Rep. Prog. Phys. 55, 241 (1992).

[17] W. Götze, J. Phys.: Condens. Matter 11, A1 (1999).

[18] D. Forster, Hydrodynamic Fluctuations, Broken Symmetry, and Correlation Functions (W.A. Benjamin, Reading, MA, 1975).

[19] G. K. Batchelor, J. Fluid Mech 83, 97 (1977).

[20] R. A. Lionberger and W. B. Russel, J. Rheol 38, 1885 (1994).

[21] V. Trappe and D. A. Weitz, Phys. Rev. Lett. 85, 449 (2000).

[22] V. Prasad, V. Trappe, A. D. Dinsmore, P. N. Segre, L. Cipelletti, and D. A. Weitz, Faraday Disc. 123, 1 (2003).

[23] J. F. Brady, J. Chem. Phys. 99, 567 (1993).

[24] M. Fuchs and M. R. Mayr, Phys. Rev. E 60, 5742 (1999).

[25] T. Franosch, W. Götze, M. R. Mayr, and A. P. Singh, J. Non-Cryst. Solids 235-237, 71 (1998).

[26] M.E. Cates, C.B. Holmes, M. Fuchs and O. Henrich, in Unifying Concepts in Granular Media and Glasses, edited by A. Coniglio et al., (Elsevier, Amsterdam, 2004), p. 203.

[27] W. Götze, Z. Phys. B 56, 139 (1984). 


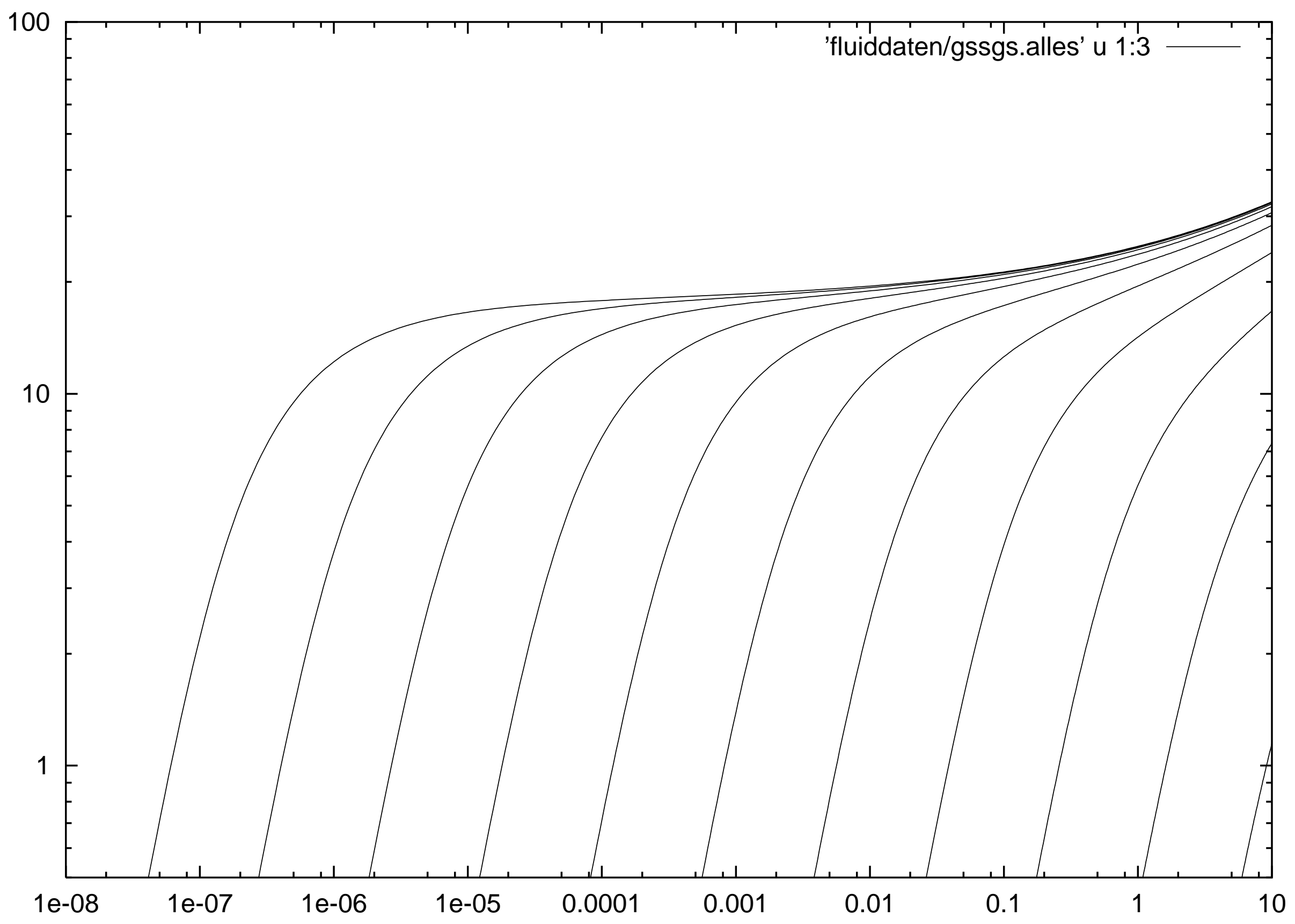




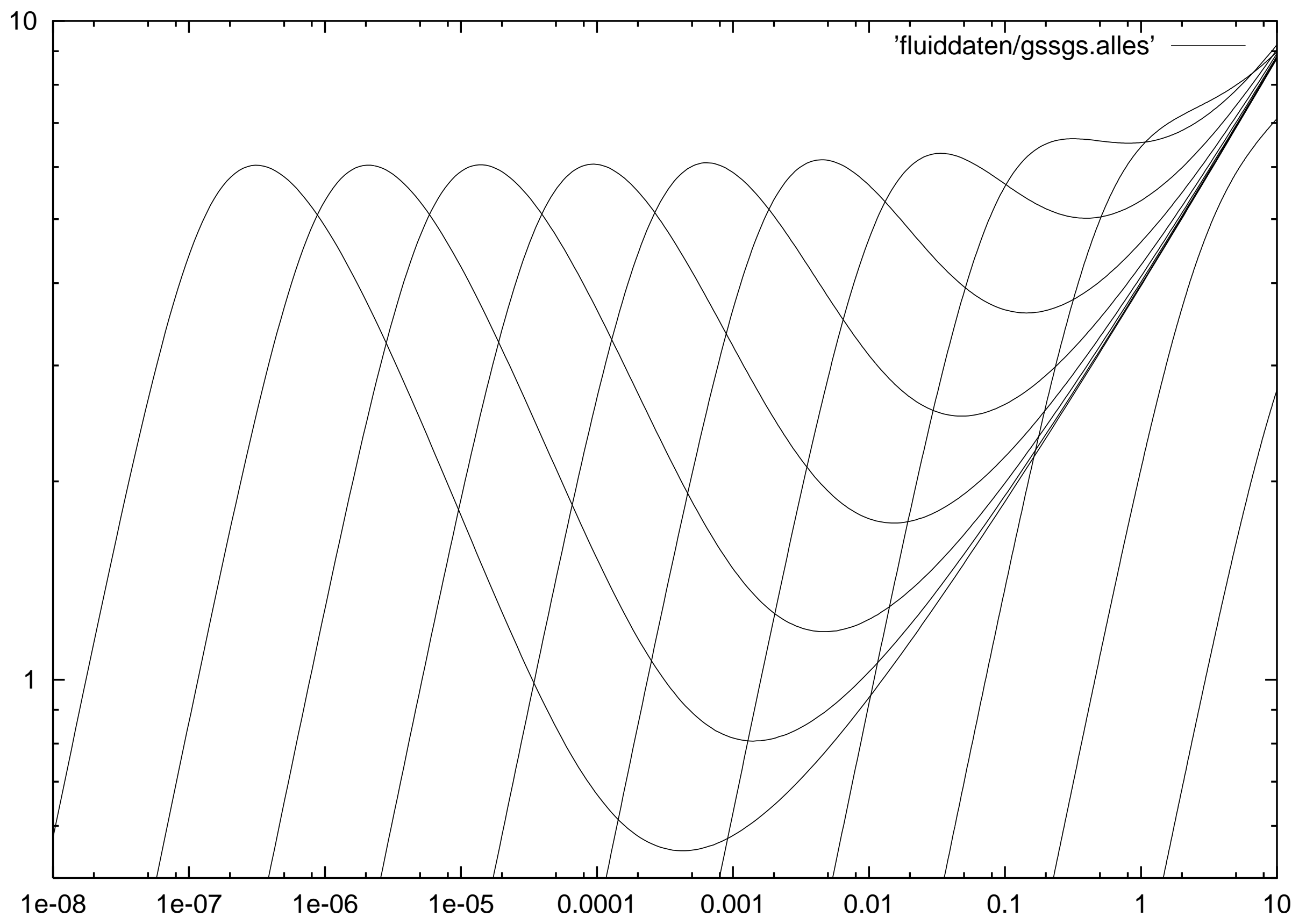

